\title{
Modeling and simulation of multi-component aerosol dynamics
}

\author{
YALCHIN EFENDIEV \\ Department of Mathematics, Texas A\&M University, College Station, TX 77843-3368 \\ E-mail: efendiev@math.tamu.edu
}

\begin{abstract}
In this paper we consider the modeling of heterogeneous aerosol coagulation where the heterogeneous aerosol particles (called droplets) contain smaller particles (enclosures). Droplets and enclosures coagulate with different collision kernels. We discuss macroscopic modeling and simulation of these processes using both deterministic and Monte-Carlo methods.
\end{abstract}

Mathematical subject classification: $65 \mathrm{C} 05,65 \mathrm{C} 35$.

Key words: multi-component, aerosol, Monte Carlo, heterogeneous.

\section{Introduction}

The study of aerosol dynamics is often limited to homogeneous, singlecomponent aerosol particles. Furthermore, even those studies that have employed more than one component assume that aerosol is a homogeneous mixture of all multi-component constituents. However, it is known that phase segregation will take place within an aerosol droplet if the thermodynamics and kinetics are favorable, in a manner analogous to that observed in bulk materials. So in order to accurately predict and control multi-component particle production it is not sufficient to assume that aerosol is a homogeneous mixture of all multicomponent constituents. In a manner analogous to bulk materials our goal is to be able to control and characterize the overall behavior of the multi-component aerosol particles.

It is becoming increasingly apparent that multi-component aerosol particles are of both industrial importance and an area in need of significant research activity. 
There have been a number of multi-component aerosol dynamics studies with heterogeneous aerosol particles that have shown the importance of multi-component aerosol particles in material synthesis. There are experimental studies on the formation of binary metal oxide systems with application to removal of heavy metals $[4,5]$ as well as the formation of materials with novel and interesting properties $[10,11,26]$. One of the main goals in this research is to study the evolution of the internal state of the aerosol droplets and accurately predict and control their internal morphology. Initial success in growing interesting microstructures [26] indicated that further research was warranted. In subsequent studies both in-situ investigation into the formation process [18], multi-component aerosol dynamic modeling [3] and molecular dynamics computation [27] have been employed. One of the primary conclusions was that for high temperatures where these materials are typically grown, nanodroplets are in liquid-like state, and that phase segregation taking place within the nanodroplet was probably limited by the transport within nanodroplet. This was one of our working assumptions in modeling and simulation [7, 89] of multi-component nanodroplets. In Figure 1 we present an example of TEM results for the $\mathrm{SiO}_{2} / \mathrm{Fe}_{2} \mathrm{O}_{3}$ system.

In the course of this paper, we shall use the terms minor phase and enclosure interchangeable to refer to the component within each aerosol droplet, and droplet or aerosol when referring to the major phase. The mathematical formulation of the problem allows one to consider the enclosures as particles inside the droplet that coagulate with collision kernel that is different from that of droplet coagulation. The temporal evolution of the aerosol and enclosures is schematically depicted in Figure 2. Instantaneous coalescence assumption is used in the modeling that is justified by experiments. More detailed description of the experiment can be found in [26].

In the paper we will be interested when collision processes governing droplet coagulation and enclosure coagulation are different. In particular, we will be interested in the macroscale quantities and their evolution. One of our main goal is to discuss the mean enclosure volume of each droplet and their asymptotic behavior. We will show that the mean enclosure volumes of each droplet tends to a constant if mean number of enclosures per droplet increases. This fact greatly simplifies the problem (reduces the degrees of freedom) and allows us to derive 


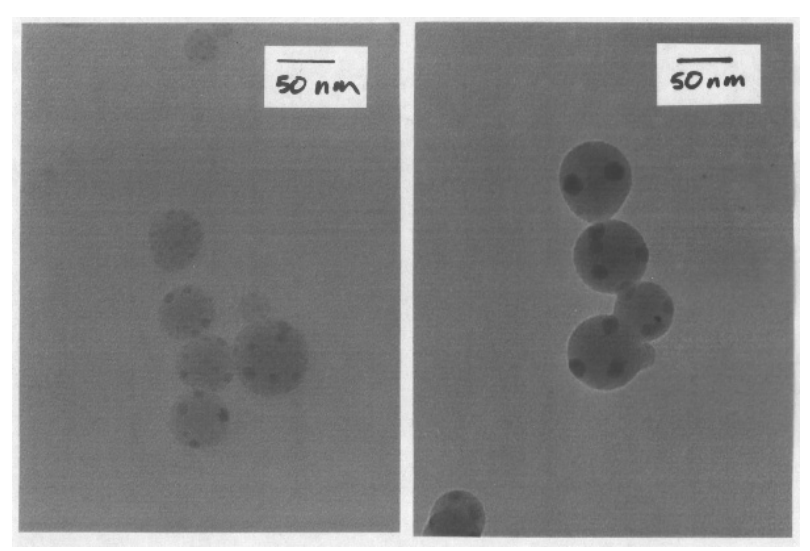

Figure 1 - Evolution of the aerosol $\left(\mathrm{SiO}_{2}\right)$ and minor phase $\left(\mathrm{Fe}_{2} \mathrm{O}_{3}\right)$ during the growth of $\mathrm{SiO}_{2} / \mathrm{Fe}_{2} \mathrm{O}_{3}$ nanocomposites.

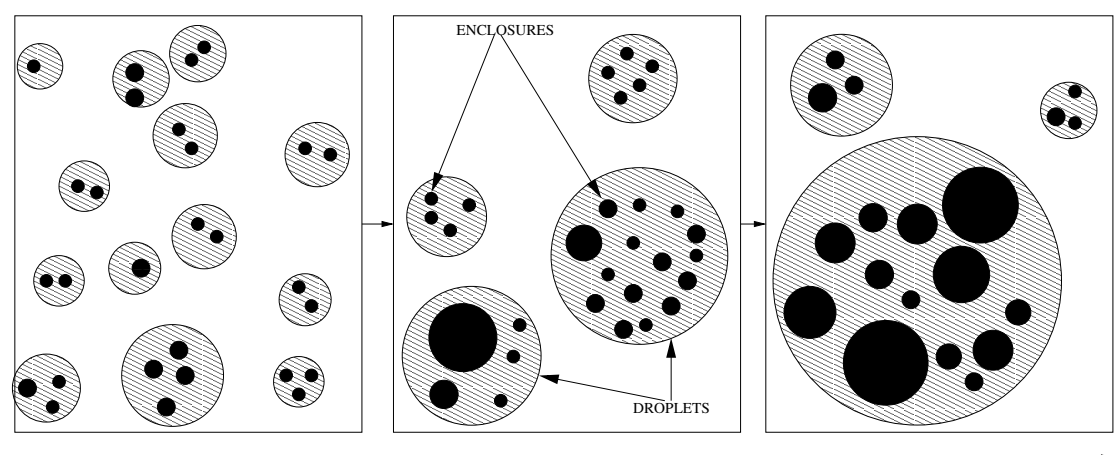

Increasing Residence Time

Figure 2 - Schematic description of droplet-enclosure growth process.

macroscale models. Our argument is not mathematically rigorous and we back it up with physical arguments as well as with Monte-Carlo simulations. The global existence result for the generalized model that describes heterogeneous aerosol coagulation is also presented.

The heterogeneous aerosol coagulation processes have multi-scale nature. In particular, because of multi-scale nature of the time and dimension scales involved in these processes we try to simplify the computations and develop more innovative numerical techniques. For the deterministic macroscale models this involves the use of coarse spaces for the discretization of the volume space. 
On the other hand, Monte Carlo methods have the advantage that multi-scale and time phenomena can be simultaneously solved without the requirement of a single unifying governing multi-variate equation. We discuss the difficulties of Monte-Carlo methods for heterogeneous aerosol coagulation processes due to the multiscale nature of these processes and the approaches to overcome these difficulties.

The paper is organized as follows. In the next section we discuss the deterministic models and the assumptions involved in this modeling. Section 3 is devoted to numerical results. The conclusions are drawn in section 4 .

\section{Heterogeneous aerosol coagulation}

\subsection{Deterministic modeling of heterogeneous aerosol coagulation}

Conceptually, there are two kinds of mathematical models describing the dynamics of homogeneous aerosol particles: deterministic and stochastic models. The deterministic models describe the evolution of some average quantities, e.g., the number density of aerosol particles with certain properties. For spherical particles, we are usually interested in the number density of the particles, $N(t, V)$, with volume $V$. More precisely, $N(t, V) d V$ is the number of aerosol particles with volumes between $V$ and $V+d V$. The coagulation process is characterized by a collision kernel which describes the collision mechanism. The expression for the collision kernel is based on a physical model and particle properties (size, density, etc). The equation for the evolution of $N(t, V)$ was first introduced by Smoluchowski [23] (survey paper [6]):

$$
\begin{aligned}
\frac{d N(t, V)}{d t}= & \frac{1}{2} \int_{0}^{V} K(U, V-U) N(t, U) N(t, V-U) d U \\
& -N(t, V) \int_{0}^{\infty} K(V, U) N(t, U) d U .
\end{aligned}
$$

The first term on the right hand side accounts for the gain of particles of volume $V$ due to the collision of particles of volume $U$ and $V-U$, and the second term on the right hand side accounts for the loss of particles due to the collision of particles of volume $V$ with any other particle of volume $U$. Smoluchowski's equation can be generalized by including additional physical effects, such as 
fragmentation, condensation, nucleation, diffusion and etc. Some of commonly used collisions kernels in aerosol coagulation ([12]) are free-molecule collision kernel

$$
K^{F}(U, V)=K_{0}\left(\frac{1}{U}+\frac{1}{V}\right)^{1 / 2}\left(U^{1 / 3}+V^{1 / 3}\right)^{2},
$$

where $K_{0}=\left(\frac{3}{4 \pi}\right)^{1 / 6}\left(\frac{6 k T}{\rho}\right)^{1 / 2}$ and Brownian (continuum regime) collision kernel

$$
K^{D}(u, v)=K_{0}\left(u^{1 / 3}+v^{1 / 3}\right)\left(\frac{1}{u^{1 / 3}}+\frac{1}{v^{1 / 3}}\right),
$$

where $K_{0}=\frac{2 k T}{3 \mu}$. Here $k$ denotes Boltzmann's constant, $T$ is the temperature, $\mu$ is the viscosity of the medium comprising aerosol particles and $\rho$ is the density of droplets. Throughout the paper we will assume that the collision kernels are homogeneous, i.e., $K(\lambda u, \lambda v)=\lambda^{p} K(u, v)$. Note that for free-molecule regime $p=1 / 6$ and for Brownian coagulation $p=0$.

For the heterogeneous aerosol coagulation we assume that the collision kernels for the enclosure coagulation (or coagulation of the minor phase) has $p=p_{e}$ and the collision kernel for the droplet coagulation has $p=p_{d}$. In particular, we are interested when the collision kernels for enclosure and droplet populations have different degrees of homogeneities $(p)$. For iron/silica oxide binary system (see [7]), the collision kernel for enclosure coagulation has $p_{e}=0$, while the collision kernel for droplet population has $p_{d}=1 / 6$.

The difficulty in mathematical modeling of the enclosure distribution of the whole system lies in the nonlinear nature of enclosure as well as in droplet coagulations. Assume that the collision kernel of the enclosures in each droplet is the same, and all droplets contain large number of enclosures so that we can describe their evolution by Smoluchowski's equation. Denoting the number density of the enclosures in a droplet with volume $V$, by $n_{V}(t, u) / V$, we can write a population balance equation for the enclosures in a droplet of volume $V$,

$$
\begin{aligned}
\frac{d n_{V}(t, v)}{d t}= & \frac{1}{2 V} \int_{0}^{v} K(u, v-u) n_{V}(t, u) n_{V}(t, v-u) d u \\
& -\frac{1}{V} n_{V}(t, v) \int_{0}^{\infty} K(v, u) n_{V}(t, u) d u .
\end{aligned}
$$


Note that $n_{V}(t, u) d u$ is the number of enclosures with volume between $u$ and $u+d u$ that are in the droplet of volume $V$. In general, in order to find the enclosure distribution for the whole system one needs to add the enclosure distributions over all droplets,

$$
n^{\text {total }}(t, u)=\int_{0}^{\infty} n_{V}(t, u) N(t, V) d V .
$$

Here we denote by $n^{\text {total }}(t, u)$, the enclosure distribution of the whole system, and $N(t, V)$ is the number density of the droplets. Since the equation (4) is nonlinear and the operation (5) is linear one cannot derive an equation for the evolution of $n^{\text {total }}(t, u)$ analytically. Moreover, it is not possible to describe the individual enclosures inside individual droplets since the numerical efforts would be tremendous.

One of approaches in modeling is to limit the description of enclosure population to their basic statistics. This idea is utilized for a simple two-component system characterizing $\mathrm{SiO}_{2} / \mathrm{Fe}_{2} \mathrm{O}_{3}$, in one of our work [7]. A goal is to model $N_{n, \bar{u}, \sigma}(t, V)$, which is the number density of aerosols with volume $V$ and $n$ enclosures whose size distribution is characterized only by their (enclosures) mean volume $\bar{u}$ and standard deviation $\sigma$. Note that here we assume that the enclosure population has log-normal distribution. This assumption will be discussed in details below. The equation for the evolution of $N_{n, \bar{u}, \sigma}(t, V)$ can be written based on conservation principles (see [7])

$$
\begin{aligned}
& \frac{d N_{n, \bar{u}, \sigma}(t, V)}{d t}=\frac{1}{2} \int_{0}^{V} K(U, V-U) \\
& \sum N_{k^{\prime}, \bar{u}^{\prime}, \sigma^{\prime}}(t, U) N_{k^{\prime \prime}, \bar{u}^{\prime \prime}, \sigma^{\prime \prime}}(t, V-U) d U \\
& \quad-N_{n, \bar{u}, \sigma}(t, V) \int_{0}^{\infty} K(U, V) \sum N_{k^{\prime}, \bar{u}^{\prime}, \sigma^{\prime}}(t, U) d U \\
& \quad+\sum \gamma_{\left(k^{\prime}, \bar{u}^{\prime}, \sigma^{\prime}\right) \rightarrow(n, \bar{u}, \sigma)}(t, V) N_{k^{\prime}, \bar{u}^{\prime}, \sigma^{\prime}}(t, V) \\
& -\sum \gamma_{(n, \bar{u}, \sigma) \rightarrow\left(k^{\prime}, \bar{u}^{\prime}, \sigma^{\prime}\right)}(t, V) N_{n, \bar{u}, \sigma}(t, V) .
\end{aligned}
$$

The first two terms in the equation (6) account for the gain and loss due to coagulation of droplets. We omit the details of the summations because they are 
cumbersome (the details in [7]). For example, in the first term the summation is taken over all possible $\left(k^{\prime}, \bar{u}^{\prime}, \sigma^{\prime}\right)$ and $\left(k^{\prime \prime}, \bar{u}^{\prime \prime}, \sigma^{\prime \prime}\right)$ such that if two droplets with volume $U$ and $V-U$ and enclosure distribution characterized by $\left(k^{\prime}, \bar{u}^{\prime}, \sigma^{\prime}\right)$ and $\left(k^{\prime \prime}, \bar{u}^{\prime \prime}, \sigma^{\prime \prime}\right)$ collide then the enclosure distribution of the resulting droplet is characterized by $(k, \bar{u}, \sigma)$. The last two terms refer to the gain and loss due to interaction of the enclosures inside a droplet of volume $V$. The quantity $\gamma_{\left(k^{\prime}, \bar{u}^{\prime}, \sigma^{\prime}\right) \rightarrow(n, \bar{u}, \sigma)}(t, V)$ denotes the rate that in a droplet of volume $V$ the distribution of enclosure population will change from $\left(k^{\prime}, \bar{u}^{\prime}, \sigma^{\prime}\right)$ to $(n, \bar{u}, \sigma)$ during the time $d t$. The details of the summation are again omitted.

In order to find $\gamma_{\left(k^{\prime}, \bar{u}^{\prime}, \sigma^{\prime}\right) \rightarrow(n, \bar{u}, \sigma)}(t, V)$ we need to model the evolution of basic statistics of the enclosure population in each droplet. The latter can be easily done by multiplying Smoluchowski's equation by $v^{i}(i=0,1,2)$ and integrating over all $v([7,16])$. This yields the following equations for the moments $d M_{0} / d t=-K\left[M_{0}^{2}+M_{1 / 3} M_{-1 / 3}\right], d M_{1} / d t=0$, and $d M_{2} / d t=$ $2 K\left[M_{1}^{2}+M_{4 / 3} M_{2 / 3}\right]$, where $M_{i}=\int v^{i} n(v, t)$. To close this system an assumption about the nature of the enclosure distribution is needed. It is known that for large times the enclosure distribution can be approximated by the log-normal distribution [16, 20, 19]. Assuming that the enclosure distribution is log-normal $M_{k}=n v_{g}^{k} \exp \left(\frac{9}{2} k^{2} \log ^{2}(\sigma)\right)$ one can derive an evolution equation for $n$ (the total number of particles), $v_{g}$ (the geometric mean particle volume), and $\sigma$ (the geometric standard deviation), from where $\gamma$ (see eq. (6)) can be computed analytically (see [7]). To solve (6) we employed binning strategy that divides the space of droplet volumes and enclosure numbers into coarse partitions [7]. The latter is necessary because the range of droplet volumes and the number of enclosures is very large which makes it difficult to use standard discretization techniques.

\subsection{Assumptions involved in deterministic modeling}

In this section we will discuss the main assumption involved in this modeling that is not addressed in [7]. This assumption is crucial for the modeling purposes and needed in order to preserve the log-normal distribution of enclosure population after two droplet collisions. After the collision of two droplets the distribution 
of enclosure population in the resulting droplet is equal to the sum of the distributions of enclosure populations in the colliding droplets. Thus, it will remain log-normal if and only if the mean volume of the enclosures and their variance in the colliding droplets are the same. We claim that the mean enclosure volume of each droplet is the same among the droplets for large times provided $p_{e}<p_{d}$. The mean enclosure volume of each droplet $i$ is defined as $\bar{u}_{i}=U_{i} / n_{i}$, where $U_{i}$ is total enclosure volume in a droplet $i$, and $n_{i}$ is the number of the enclosures in the droplet $i$. This fact (the equality of mean enclosure volumes) plays an important role in modeling of aerosol systems where the enclosure population is characterized only with its basic statistics (e.g., total number, mean and variance). Furthermore, this assumption greatly reduces the number of unknowns of the problem and can be used for various simple models. To show the validity of this assumption we will need to discuss the following two facts (1) the equality of the enclosure concentration in each droplet for large times (2) the behavior of the mean enclosure numbers in each droplet. With these two results we can argue that the mean enclosure volume of each droplet is the same among the droplets for large times provided the mean number of enclosures increases. Note that the latter is true if $p_{e}<p_{d}$.

\section{The equality of the enclosure concentration in each droplet at large times.}

Define the concentration of enclosures in a droplet $i$ by $c_{i}$, which is given as the total enclosure volume in this droplet divided by the droplet volume. We assert that $c_{i}$ is independent of $i, c_{i}=c$. This assumption is true if initially the concentration of the enclosures in each droplet is uniform. Then these concentrations will remain constant. Indeed, if two droplets with volumes $V_{1}$ and $V_{2}$ collide, then the total concentration of enclosures in a resulting droplet with volume $V_{1}+V_{2}$ will be $c\left(V_{1}+V_{2}\right)$, i.e., concentration will remain constant equal to $c$. It can be shown that if the enclosure concentration in droplets are not the same initially it will evolve to the state when enclosure concentrations will become equal. This fact has been observed numerically in our Monte Carlo simulations and can be studied independently of the size distribution of enclosure population. Indeed, introduce the quantity $N(t, V, c)$, number density of droplets with volume $V$ and with the enclosure concentration $c$. Note that if two droplets 
with volume $V_{1}$ and $V_{2}$, and enclosure concentrations $c_{1}$ and $c_{2}$ collide, then the volume of the resulting droplet is $V=V_{1}+V_{2}$, and the enclosure concentration is $c=\left(c_{1} V_{1}+c_{2} V_{2}\right) / V$. Thus the evolution of $N(t, V, c)$ is simply governed by

$$
\begin{gathered}
\frac{d N(t, V, c)}{d t}=\frac{1}{2} \int_{0}^{V} K(U, V-U) \\
\int_{0}^{c} N(t, U, c 1) N\left(t, U, \frac{c V-c_{1} U}{V-U}\right) d U d c_{1} \\
-N(t, V) \int_{0}^{\infty} \int_{0}^{\infty} K(V, U) N(t, U, c 1) d U d c_{1} .
\end{gathered}
$$

Here the first term accounts for the gain of particles with volume $V$ and the enclosure concentration $c$. These particles are formed as a result of a collision of droplets with volume $U$ and $V-U$ and enclosure concentrations $c 1$ and $\left(c V-c_{1} U\right) /(V-U)$. Similarly, the second term accounts for the loss of droplets with volume $V$ and the enclosure concentration $c$. The asymptotics of these kinds of equations have been studied in [13,21]. It was shown that $\int_{0}^{\infty} \int_{0}^{\infty}(c-\bar{c}) V N(t, V, c) d V d c$ converges to zero as $t \rightarrow \infty$. We see from (7) that the study of the concentration of enclosure population does not depend on the distribution of enclosure population in each droplet. This is a fundamental difference between this study and the study of mean number of enclosures per droplet as well as the study of mean enclosure volume of each droplet.

The behavior of the mean enclosure numbers in each droplet. The assumption of the equality of mean volume of enclosures in each droplet, $u_{i}$, holds if $p_{e}<p_{d}$, and it is approximate if $p_{e} \approx p_{d}$. We do not have rigorous mathematical proof of this fact, and will use physical argument as well as Monte-Carlo simulations to demonstrate this. For this reason, we introduce the mean number of enclosures per droplet, i.e., $n_{t o t} / M_{\text {tot }}$, where $n_{t o t}$ is the total number of enclosures, and $M_{t o t}$ is the total number of droplets. If we assume $p_{e}<p_{d}$ it can be shown that the mean number of enclosures per droplet increases. Indeed, using the self-similar theories (see [1]) it can be shown that in each droplet the number of enclosures decays as $t^{-1 /\left(1-p_{e}\right)}$. This rate is the same for all the enclosures, consequently the total number of enclosures decays as $t^{-1 /\left(1-p_{e}\right)}$. On the other hand, the total number of droplets decays as $t^{-1 /\left(1-p_{d}\right)}$. Consequently 
mean number of enclosures per droplet grows as $t^{-1 /\left(1-p_{e}\right)+1 /\left(1-p_{d}\right)}$. Thus if $p_{e}<p_{d}$ the mean number of enclosures in each droplet will increase as $t^{\gamma}$, $\gamma=-1 /\left(1-p_{e}\right)+1 /\left(1-p_{d}\right)>0$. We will confirm this rate in our MonteCarlo computations.

Next we present our argument on $u_{i}$. The characteristic interaction (i.e., coagulation) time for the enclosures in each droplet $V_{i}$, is given by

$$
t_{i}^{c}=K_{0}^{e} \frac{V_{i}}{n_{i}},
$$

where $n_{i}$ are the number of enclosures in a droplet $i$ and $K_{0}^{e}$ is a constant that depends on the physical parameters (the background media). We assume that at an asymptotic condition, the characteristic interaction times for the enclosures in each droplet are balanced with the characteristic coagulation time for the droplets. Consequently, the characteristic interaction time for the enclosures is the same. Since the total enclosure volume of a droplet with volume $V_{i}$, is $c V_{i}$, $t_{i}^{c}$ can be written as

$$
t_{i}^{c}=K_{0}^{e} \frac{\bar{u}_{i}}{c},
$$

where $\bar{u}_{i}$ is the mean volume of the enclosures of the droplet. So at an asymptotic limit we expect the mean enclosure volumes of each droplet to be constant. However, this characteristic time (8) is only defined if $n_{i}>1$. So if the number of enclosures per droplet is close to unity the characteristic enclosure coagulation times (defined in (8)) in each droplet will in general not be equal to each other and we need to take account of the variation in number of enclosures per droplets. This corresponds to the case when $p_{e} \geq p_{d}$. To see this we consider the case when there is one enclosure per droplet. Then the volume of the enclosure (or mean volume of the enclosures per droplet) is equal to $c V$, where $c$ is the total concentration independent of the droplets. Thus mean volumes of the enclosures will have the same distribution as the volume of droplets. Volume of droplets has self-preserving shape distribution with nonzero width on the log scale (e.g. [12]). From here we can conclude that mean volume of enclosures in a droplet is not the same if the mean number of enclosures per droplet is small. We can also show that the standard deviations of the enclosure populations in each droplet will be 
constant (independent of the droplet) for large times if the mean enclosure volume of each droplet is constant. Consider, $S_{2}=\sum\left(v_{i}-\bar{v}\right)^{2} n\left(v_{i}, t\right)=M_{2}-\bar{v}^{2} n$, where $M_{2}=\sum v_{i}^{2} n\left(v_{i}, t\right) d v, n\left(v_{i}, t\right)$ is the number of enclosure with volume $v_{i}$, $n$ is the total number of enclosures, and $\bar{v}$ is the mean enclosure volume. For two colliding droplets with enclosure distribution $n^{\prime}(v, t)$ and $n^{\prime \prime}(v, t)$ we have $M_{2}=$ $M_{2}^{\prime}+M_{2}^{\prime \prime}$. From where assuming that $\bar{v}^{\prime}=\bar{v}^{\prime \prime}$ we have $S_{2}=S_{2}^{\prime}+S_{2}^{\prime \prime}, n=n^{\prime}+n^{\prime \prime}$. Then for standard deviation $s=S_{2} / n$ we have $s=\left(n^{\prime} S_{2}^{\prime}+n^{\prime \prime} S_{2}^{\prime \prime}\right) /\left(n^{\prime}+n^{\prime \prime}\right)$. From here using argument similar to the analysis of the enclosure concentration we can show that $s$ tends to a constant for large times. The rigorous proof of this fact is a subject of future study.

\section{Numerical methods and results}

\subsection{Monte-Carlo methods}

Monte Carlo methods to simulate particulate growth processes are not new, and the theoretical foundations have been discussed extensively in the literature $[14,24,25]$. Basically the Monte Carlo approach utilizes probabilistic tools to study a finite dimensional subsystem in order to infer the properties of the whole system. Here we briefly discuss Monte-Carlo methods used in our simulations.

There are in general two types of finite-volume Monte-Carlo techniques. In the first approach, the user sets the time interval $\Delta t$, and uses Monte-Carlo to decide which and how many events will be realized. This method is sometimes referred to as time driven Monte-Carlo. In the second approach, the user selects a single event and then advances the time by an appropriate increment. In the method presented here we employ the first method for the enclosures or minor phase, and the second method to describe the droplets/aerosol. More precisely, we first select a single coagulation event for the droplets, and compute the time $\Delta T$ required for this event. Then for each droplet we calculate the enclosure interactions occurred during this time interval.

At each step of the simulation, droplets $i$ with volume $V_{i}$ and $j$ with volume $V_{j}$ are selected to coagulate, and a new droplet of size $V_{i}+V_{j}$ is formed with a probability that is proportional to the coagulation probability, $K_{i j}$. To calculate the mean inter-event time between two successive events we consider a system 
with initial number concentration $C_{0}$ and total number $N_{0}$ droplets in the simulation. Then as outlined by Smith and Matsoukas [22] the effective real volume being simulated is $N_{0} / C_{0}$, so that one coagulation event in our (model) system represents $C_{0} / N_{0}$ actual droplets per unit volume. To connect our simulations to real time we calculate the inter-event time, by noting that the time between two events is inversely proportional to the sum of the rates of all possible events. If for example $k$ successful events are realized, then the remaining number of droplets in the system is $N_{k}=N-k$, and the total number concentration of the system $C_{k}$ is given by $\frac{C_{k}}{C_{0}}=\frac{N_{k}}{N_{0}}$. The mean inter-event time after $k$ coagulations as [22]

$$
\Delta T_{k}=\frac{2 N_{0}}{C_{0} \sum_{i=1}^{N_{k-1}} \sum_{j=1}^{N_{k-1}} K_{i j}^{F}} .
$$

For each droplet we use the inter-event time to determine the number of successful enclosure interactions (coagulation driven growth) before the next droplet coagulation. This unfortunately restricts the time step, because modeling the internal state of the droplets requires complete knowledge of enclosure distribution within each droplet.

In an analogous manner to that of the droplets we also define the mean interevent time for the enclosures in a droplet of volume $V$ as

$$
\Delta t=\frac{2 V}{\sum_{i=1}^{n-1} \sum_{j=1}^{n-1} K_{i j}^{D}},
$$

where $n$ is the number of the enclosures, and $n / V$ is their number density. The number of successful enclosure interactions inside the droplet during the time interval $\Delta T_{1}$ is given by the integer $k$ which satisfies

$$
\begin{aligned}
\sum_{i=1}^{k} & \frac{2 V}{\left\langle K_{i j}^{D}\right\rangle(n-i)(n-i-1)} \leq \Delta T_{1} \\
& \leq \sum_{i=1}^{k+1} \frac{2 V}{\left\langle K_{i j}^{D}\right\rangle(n-i)(n-i-1)} .
\end{aligned}
$$

On the left hand side of (10) we have the total time needed for the coagulation of $k$ enclosures and on the right hand side the total time needed for the coagulation of $k+1$ enclosures. 
When the number of droplets drops to half the initial value, we replicate the droplets and their internal state. In order to preserve the physical connection to real time, the topping up process must preserve the average behavior of the system corresponding to the time prior to topping up. In particular, one has to ensure that the characteristic time for droplet collisions stays the same, and to do this requires an increase in the system volume in proportion to the increase in droplets. On the other hand, if the number of enclosures in a droplet becomes too large for the simulation, one can truncate the enclosure system within a droplet by randomly picking a certain number of enclosures and adjusting the corresponding computational volume. A flow chart of our Monte Carlo algorithm is depicted in Figure 3.

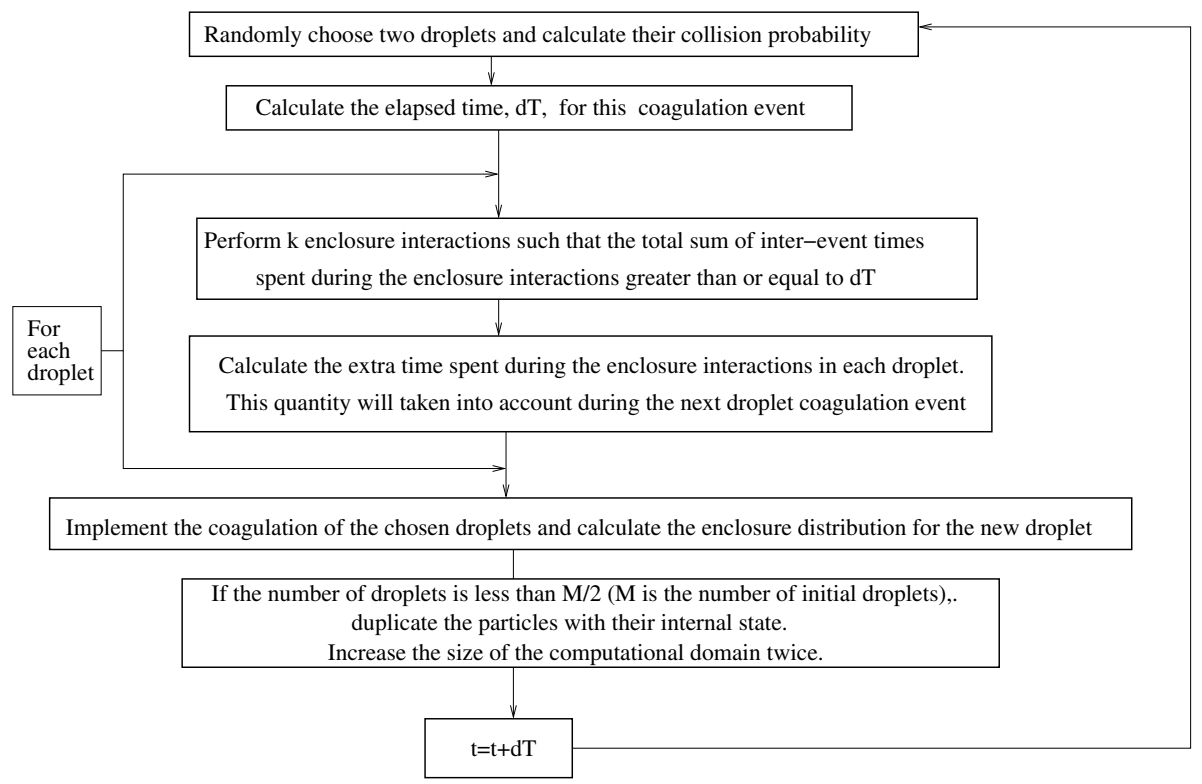

Figure 3 - Flow chart of Monte Carlo algorithm.

\subsection{Numerical results}

In the numerical examples we will use different constants $K_{0}$ for enclosure coagulation, $K_{0}^{e}$ and for droplet coagulation $K_{0}^{d}$ (see e.g. (3)). In the first numerical example we assume that enclosures coagulate in the Brownian regime 
$p_{e}=0$, while droplets coagulate with constant collision kernel $p_{d}=0$, and take $K_{0}^{e}=4.8 e-18, K_{0}^{d}=3.6 e-9, \phi=5.2 e-7$, where $\phi$ is the total concentration of the droplets (see [9]). In this case our assumption on the mean volume of the enclosures will not be exact as the number of the enclosures per droplet does not increase. In the Figure 4 we plot mean number of enclosures per droplet using our Monte-Carlo simulations. As we see from this figure that mean number of enclosures per droplet reaches constant as it was predicted since $\gamma=0$. The experiments are performed for three different values of $M$ (initial number of droplets) and $m$ (initial number of enclosures in each droplet). Because the mean number of enclosures per droplet does not increase we do not expect our assumption on $\bar{u}_{i}$ (mean enclosure volume of each droplet) discussed in the previous section to hold. Here $\bar{u}_{i}, i=1, \ldots, M$ denote the mean enclosure volume in the $i$ th droplet, and $M$ is the total number of droplets. To measure the variability of $\bar{u}_{i}$ we introduce the normalized variance $\bar{u}_{m}^{2} / \bar{u}_{m}^{2}$, where

$$
\overline{u_{m}^{2}}=\frac{1}{\sum_{i} m_{i}} \sum_{i} m_{i} \bar{u}_{i}^{2}, \quad \bar{u}_{m}=\frac{1}{\sum_{i} m_{i}} \sum_{i} m_{i} \bar{u}_{i},
$$

$m_{i}$ is the number of the mean enclosure volumes with volume $\bar{u}_{i}$. Note that the normalized variance is 1 if all $\bar{u}_{i}$ are equal, i.e., for uniform $\bar{u}_{i}$. In Figure 5 we plot the normalized variance of mean enclosure volumes of each droplet, $\bar{u}_{1}, \ldots, \bar{u}_{M}$ (in this case $(1000,3000)$ is chosen). Note that we do not expect to see significant changes for different values of $(M, m)$. The first interesting observation is that the normalized variance of the mean enclosure volumes of each droplet reaches an asymptotic value, which is quite sensitive to the mean number of particles. This asymptotic value of the variance is close to one if the mean number of enclosures per droplet is large. Indeed, if the mean number of enclosures of each droplet is large our assumption regarding of the equality of mean enclosure volumes holds. Thus, the normalized variance of this quantity will be close to one. On the other hand, when the mean number of enclosures per droplet is only one or two then the mean enclosure volumes of each droplet simply track the droplet volumes, i.e., reach the self-preserving size distribution corresponding to the Brownian regime, which is 2 . This is not difficult to check. If there is only one enclosure per droplet and assuming that the enclosure concentrations are uniform across the droplets and given by $c$, then the volume of the enclosure 
in the droplet $i$ is given by $c V_{i}$, where $V_{i}$ are the volumes of the droplets. The coagulation of the droplets is independent of the enclosures and it is known that the normalized variance of the droplet volumes is almost 2, [12].

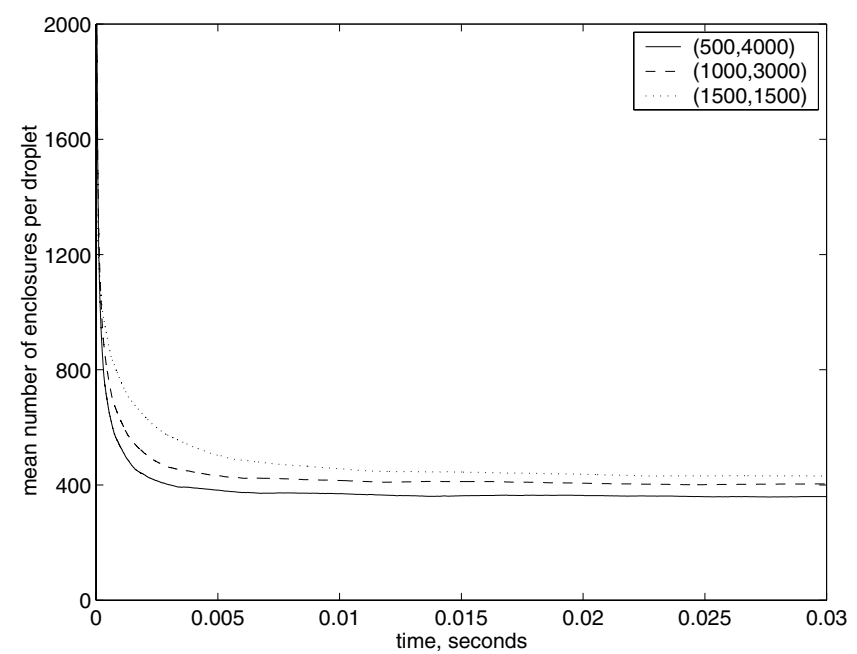

Figure 4-Mean number of the enclosures per droplet ( $\left.\frac{\text { the total number of the enclosures }}{\text { total number of droplets }}\right)$ for three values of $(M, m)$, where $M$ refers to the initial number of the droplets and $m$ refers to the initial number of the enclosures per droplet in our simulation. All droplets and enclosures are initially monodisperse. $n$ designates mean number of enclosures per droplet. Case $p_{e}=p_{d}$.

Our next set of numerical results describe the situation where the enclosures coagulate in the Brownian regime (3) $p_{d}=0$, while droplets coagulate in the free-molecular regime (2), $p_{e}=1 / 6$. We consider growth at two temperatures $2300 \mathrm{~K}$ and $2600 \mathrm{~K}$, both of which were operating conditions for experiments. The viscosity of the droplets will govern the rate of Brownian transport of the minor phase and therefore the growth rate of enclosures. The viscosity of the major component silica $\left(\mathrm{SiO}_{2}\right)$ as a function of temperature is given by [15] $\mu=10^{-8.6625(1-3556.03 \mathrm{~K} / \mathrm{T})}(\mathrm{kg}) /(\mathrm{m} \mathrm{s})$. and the density of $\mathrm{Si}_{2}$ is held constant at $\rho=5.5 \mathrm{~g} / \mathrm{cm}^{3}$. With these constants $K_{0}^{e}$ and $K_{0}^{d}$ can be easily determined.

In Figure 6 we plot mean number of enclosures per droplet for three different initial conditions. As we observe mean number of enclosures per droplet increases and the increase rate is given by $t^{1 /\left(1-p_{d}\right)-1 /\left(1-p_{e}\right)}=t^{1 / 5}$. To confirm 


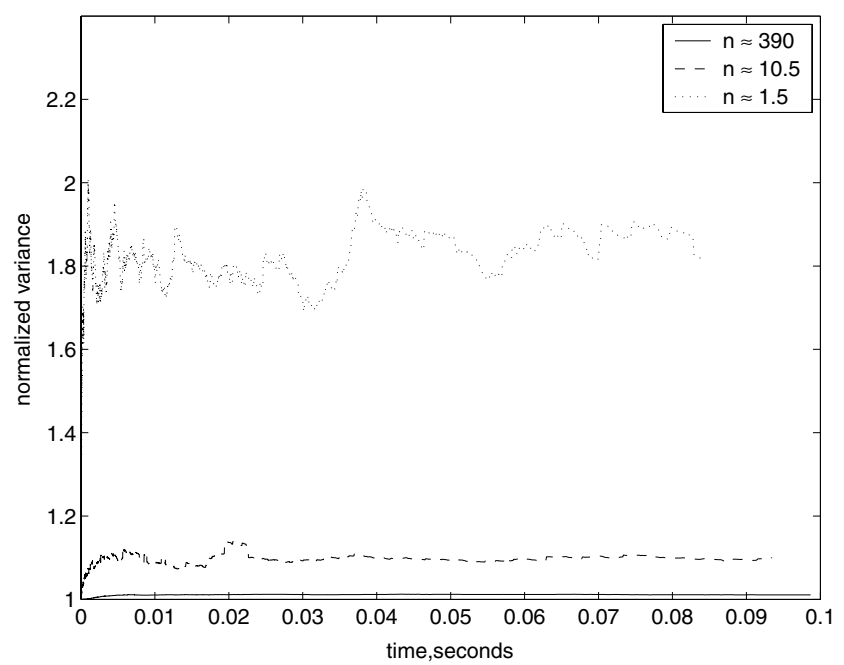

Figure 5 - The normalized variance $\left(\overline{u_{m}^{2}} / \bar{u}_{m}^{2}\right)$ of the mean enclosure volumes of each droplet for different mean number of enclosures per droplet. Case $p_{e}=p_{d}$.

this rate in Figure 7 we plot the mean number of enclosures per droplet in a $\log -\log$ scale along with a straight line with a slope $1 / 5$. It is clear from this Figure that the growth of the mean number of enclosures per droplet has a rate $1 / 5$. Since mean number of enclosures per droplet increases we expect that the mean enclosure volumes of each droplet will become uniform across droplet populations, i.e., $\bar{u}_{1}=\cdots=\bar{u}_{M}$, where $\bar{u}_{i}$ is the mean enclosure volume of $i$ th droplet and $M$ is the number of the droplets. Indeed, Figure 8 shows this. In this Figure we plot the normalized variance of mean enclosure volumes of each droplet. Clearly, this number is close to one for large times (compare with the case $p_{e}=p_{d}$, Figure 5). We note that the constants $K_{0}^{e}$ and $K_{0}^{d}$ (which depend on physical parameters) do not affect growth rate.

Finally in Figure 9 we present a comparison of Monte-Carlo computation with our deterministic model. The agreement is quite reasonable. Thus we can conclude that assumptions involved in macroscale modeling ([7]) are valid in an asymptotic regime, and the macroscopic model describes the physical process with a reasonable accuracy. In Appendix A we provide the global existence result for this model equations in a discrete case. 


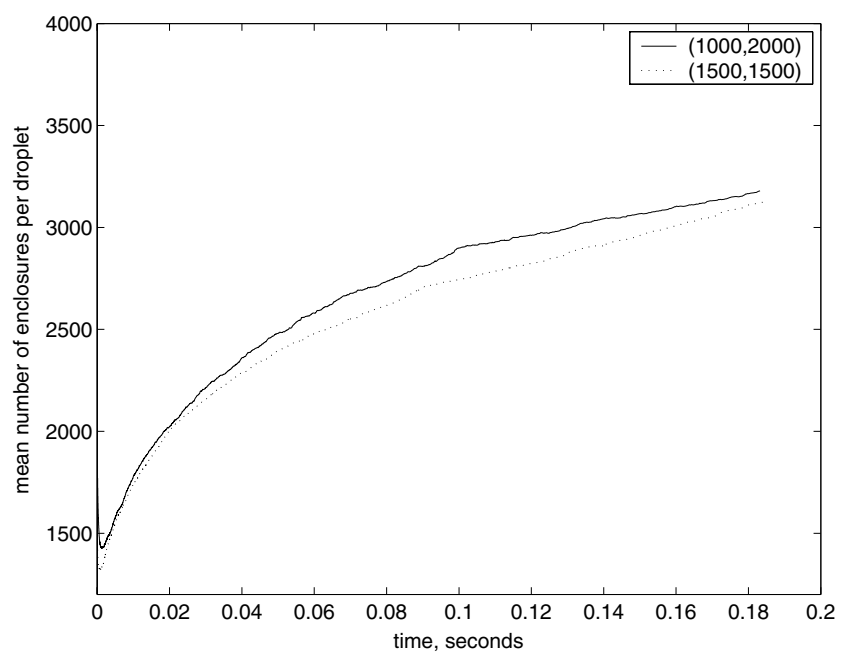

Figure 6-Mean number of the enclosures per droplet ( $\left.\frac{\text { the total number of the enclosures }}{\text { total number of droplets }}\right)$ for two values of $(M, m)$, where $M$ refers to the initial number of the droplets and $m$ refers to the initial number of the enclosures per droplet in our simulation. All droplets and enclosures are initially monodisperse. Case $p_{e}=0, p_{d}=1 / 6$.

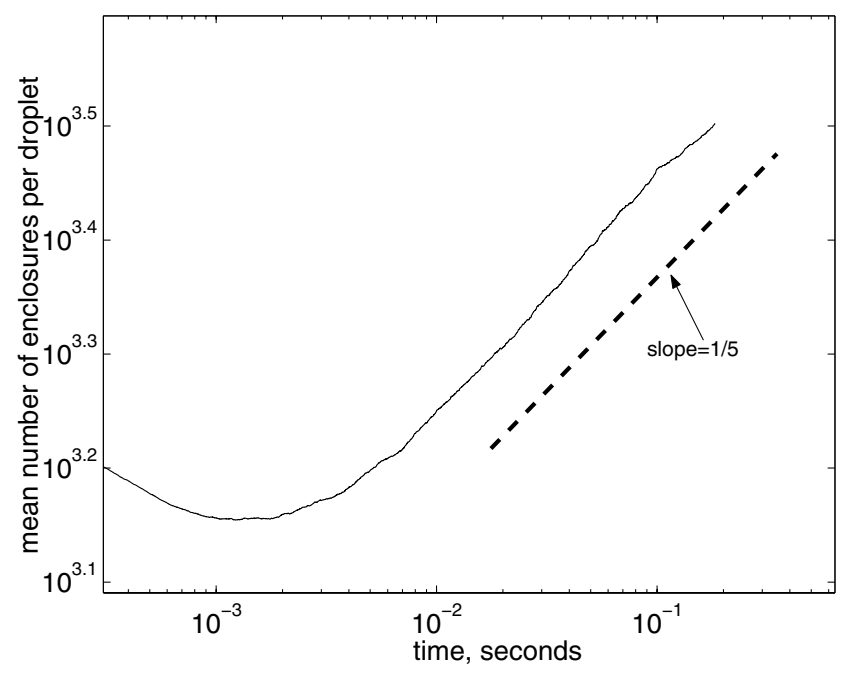

Figure 7 - Relative growth rate for different $k . \alpha=1$. The asymptote curve (designated by dashed line) grows as $\sim t^{1 / 5}, t$ is time. Case $p_{e}=0, p_{d}=1 / 6$. 


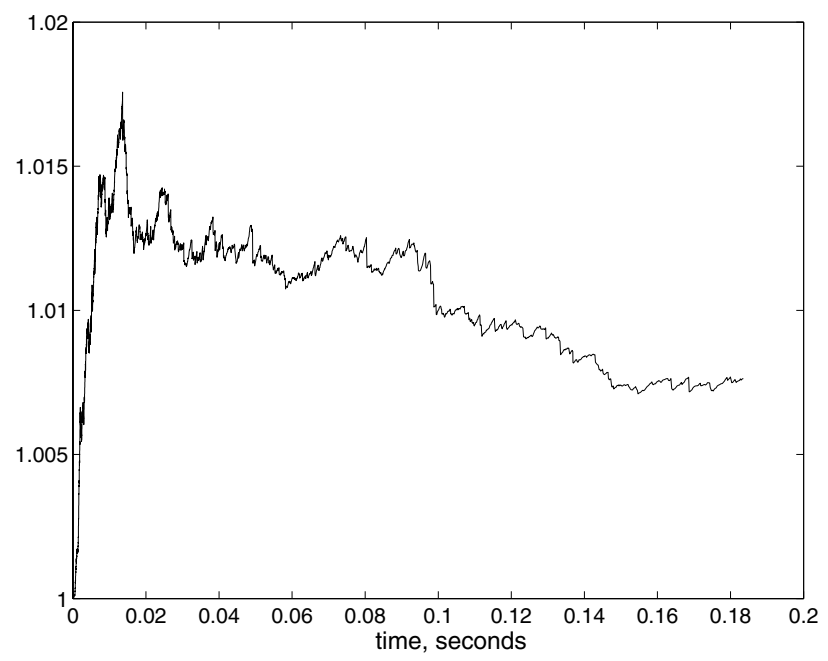

Figure 8 - The normalized variance $\left(\overline{u_{m}^{2}} / \bar{u}_{m}^{2}\right)$ of the mean enclosure volumes of each droplet. Case $p_{e}=0, p_{d}=1 / 6$.

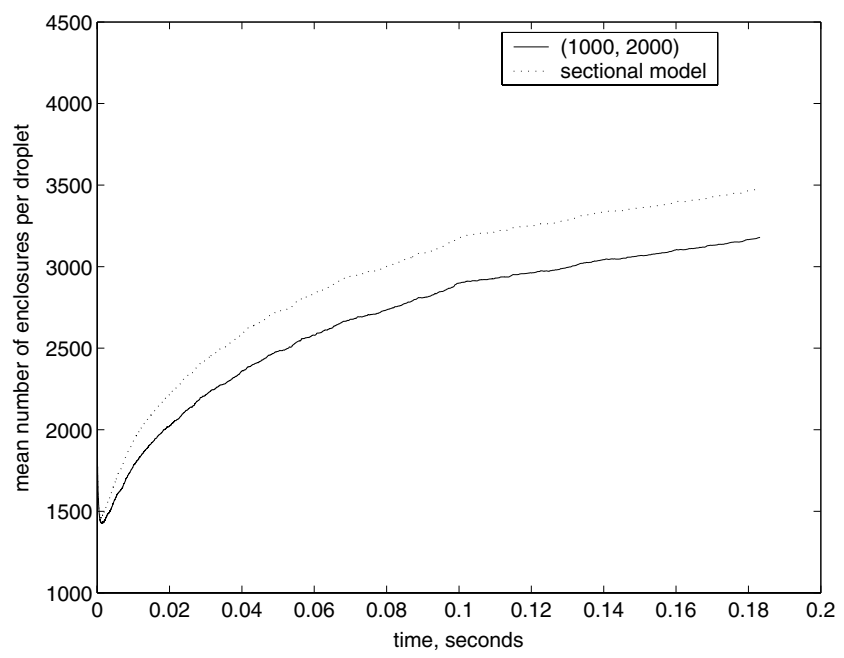

Figure 9-Mean number of the enclosures per droplet $\left(\frac{\text { the total number of the enclosures }}{\text { total number of droplets }}\right)$ compared with sectional model. 
One of the difficulties in Monte-Carlo simulations is the handling of the large number of enclosures within the droplets. Since we need to keep the number of droplets at some reasonable level, the increase in the number of enclosures per droplet makes our computations very difficult. Currently we are working on the approaches where the enclosure population in each droplet is carried only with three main statistics, the number of enclosure, the mean volume, and the variance. Each time when the detail enclosure population is needed we generate it from the log-normal distribution. If two droplets collide then the enclosure population is generated and a new droplet with whole new enclosure population is created. We carry full (detail) enclosure population for this droplet for some time until it relaxes to log-normal distribution. Then the enclosure population can be characterized with three moments. Our initial results look promising and the further research into the mathematical and computational aspects of this kind methods will be carried out.

\section{Concluding remarks}

In this paper we study the coagulation of heterogeneous aerosol particles and discuss main assumptions involved in deterministic modeling. In particular, we show that the mean volume of the enclosures per droplet is uniform for large times. The latter is crucial for the understanding of the heterogeneous coagulation processes. Because of multi-scale nature of the heterogeneous aerosol coagulation processes some innovative numerical methods are needed. We discuss our current research on this direction.

\section{A Global existence result for generalized Smoluchowski's equation}

We will consider a discrete coagulation model corresponding to the generalized Smoluchowski's equation

$$
\frac{d x_{j m}}{d t}=\frac{1}{2} \sum_{p+q=j, s+r=m} K_{p q} x_{p s} x_{q r}-\sum_{p=1, r=1}^{\infty} K_{j p} x_{j m} x_{p r}+\sum_{s=m}^{\infty} \frac{A_{m s}}{j} x_{j s} .
$$

Here $x_{i j}$ is the number of droplets whose volume $i$ and contains $j$ enclosures. We assume that $x_{j m}(0)=c_{j m}$, such that $\sum j^{2} c_{j m} \leq C$. Assume $K_{i j} \leq i^{\alpha}+j^{\alpha}$, 
$\alpha<1$, and $A_{i j} \leq C$. Finite dimensional version of the equation (11) can be obtained through truncation up to $N$ terms. The kernel is replaced by $K_{i j}^{(N)}$ as

$$
\begin{aligned}
& K_{i j}^{(N)}=\left\{K_{i j}, \text { if } i+j \leq N ; 0 \text { otherwise }\right\}, \\
& A_{i j}^{(N)}=\left\{A_{i j}, \text { if } i \leq N, j \leq N ; 0 \text { otherwise }\right\} .
\end{aligned}
$$

The finite dimensional version of (11) is

$$
\begin{aligned}
\frac{d x_{j m}^{(N)}}{d t}= & \frac{1}{2} \sum_{p+q=j, s+r=m} K_{p q}^{(N)} x_{p s}^{(N)} x_{q r}^{(N)} \\
& -\sum_{p=1, r=1}^{N} K_{j p}^{(N)} x_{j m}^{(N)} x_{p r}^{(N)}+\sum_{s=m}^{N} \frac{A_{m s}^{(N)}}{j} x_{j s}^{(N)} .
\end{aligned}
$$

Next we introduce

$$
M_{e}=\sum_{j, m} x_{j m}, \quad M_{v}=\sum_{j, m} j x_{j m}, \quad M_{e}^{(N)}=\sum_{j, m} x_{j m}^{(N)}, \quad M_{v}^{(N)}=\sum_{j, m} j x_{j m}^{(N)} .
$$

Lemma A.1. $x_{j m}^{(N)}$ exist, unique and $x_{j m}^{N}$ are positive.

Since the r.h.s. of (12) satisfies Lipschitz condition the solution exists, unique and bounded. Positiveness of the solution can be obtained similar to [17,24].

The following can be checked directly

\section{Lemma A.2.}

$$
\frac{d M_{v}^{(N)}}{d t} \leq 0, \quad \frac{d M_{e}^{(N)}}{d t} \leq 0 .
$$

Then we have

$$
\begin{aligned}
x_{j m}^{(N)} & \leq \sum_{l, p} l x_{l p}^{(N)}=M_{v}^{(N)}(t)<C,\left|d x_{j m}^{(N)} / d t\right| \\
& \leq \frac{1}{2} \sum_{p, q, s, r}(p+q) x_{p s}^{(N)} x_{q r}^{(N)}+\sum_{p, r}^{N}(j+p) x_{j m}^{(N)} x_{p r}^{(N)}+\sum_{s=m}^{N} \frac{A_{m s}^{(N)}}{j} x_{j s}^{(N)} \\
& \leq C \sum_{p, s} p x_{p s}^{(N)} \sum_{q, r} q x_{q r}^{(N)}+\sum_{j, s} x_{j s} \leq C .
\end{aligned}
$$


From here using Ascoli's lemma we obtain that $x_{j m}^{(N)}$ converges along a subsequence $N_{k}$ uniformly in any time interval. Denote $x_{j m}=\lim _{N \rightarrow \infty} x_{j m}^{(N)}$ for the subsequence. Note that $x_{j m}$ has bounded first moments because $x_{j m}^{(N)}$ has bounded first moments independent of $N$. To show that $x_{j m}$ satisfies equation (11) we need to show that

$$
\begin{aligned}
& \sum_{p} K_{j p}^{(N)} x_{p m}^{(N)} \rightarrow \sum_{p} K_{j p} x_{p m}, \quad N \rightarrow \infty \\
& \sum_{p} A_{m s}^{(N)} x_{j s}^{(N)} \rightarrow \sum_{p} A_{m s} x_{j s} \quad N \rightarrow \infty .
\end{aligned}
$$

Consider

$$
\begin{aligned}
& \left|\sum_{p} K_{j p} x_{p m}-\sum_{p} K_{j p}^{(N)} x_{p m}^{(N)}\right| \leq \sum_{p<N_{1}}\left|K_{j p} x_{p m}-K_{j p}^{(N)} x_{p m}^{(N)}\right| \\
& \quad+\sum_{p>N_{1}} K_{j p} x_{p m}+\sum_{p>N_{1}} K_{j p}^{(N)} x_{p m}^{(N)} .
\end{aligned}
$$

The first term can be made small by choosing $N$ large enough, the second (and third term) can be made small by choosing $N_{1}$ large enough in the following way:

$$
\begin{aligned}
\sum_{p>N_{1}} K_{j p} x_{p m} & \leq \sum_{p>N_{1}} j^{\alpha} x_{p m}+\sum_{p>N_{1}} p^{\alpha} x_{p m} \\
& \leq j^{\alpha} N_{1}^{-1} \sum_{p>N_{1}} p x_{p m}+N_{1}^{\alpha-1} \sum_{p>N_{1}} p x_{p m} \\
& \leq j^{\alpha} N_{1}^{-1} M_{v}(t)+N_{1}^{\alpha-1} M_{v}(t) \rightarrow 0, \quad \forall j, m .
\end{aligned}
$$

Furthermore,

$$
\begin{aligned}
& \left|\sum_{s} A_{m s} x_{j s}-\sum_{s} A_{m s}^{(N)} x_{j s}^{(N)}\right| \leq \sum_{s<N_{1}}\left|A_{m s} x_{j s}-A_{m s}^{(N)} x_{j s}^{(N)}\right| \\
& +\sum_{s>N_{1}} A_{m s} x_{j s}+\sum_{p>N_{1}} A_{m s}^{(N)} x_{j s}^{(N)}
\end{aligned}
$$


Choosing $N$ large we make the first term small. Choosing $N_{1}$ large such that $N_{1} \gg m$ we can make the second and third terms small.

Uniqueness of the solution can be obtained in a manner analogous to [2].

\section{REFERENCES}

[1] D.J. Aldous, Deterministic and stochastic models for coalescence (aggregation and coagulation): a review of the mean-field theory for probabilists, Bernoulli, 5 (1999), 3-48.

[2] J.M. Ball and J. Carr, The discrete coagulation-fragmentation equations: existence, uniqueness, and density conservation, J. Statist. Phys., 61 (1990), 203-234.

[3] P. Biswas, C.Wu, M.R. Zachariah and B.K. McMillen, Vapor phase growth of iron oxide-silica nanocomposite; Part II: Comparison of a discrete-sectional model predictions to experimental data, J. Mat. Res, (1997), 714.

[4] P. Biswas, G. Yang and M.R. Zachariah, In situ processing offerroelectric materials from lead streams by injection of gas-phase titanium precursors, Combustion Science and Technology, (1998), 183.

[5] P. Biswas and M.R. Zachariah, In situ immobilization of lead species in combustion environments by injection of gas phase silica sorbent precursors, Env. Sci. Tech., (1997), 2455.

[6] R. Drake, A general survey of teh coagulation equation. in g.m. hidy and j.r. brock, editors, Topics in current aerosol research, (1972), 201-376.

[7] Y. Efendiev, M. Luskin, H. Struchtrup and M.R. Zachariah, A hybrid sectional-moment model for coagulation and phase segregation in binary liquid nanodroplets, J. Nanoparticle Res., 4 (2002), 61-72.

[8] Y. Efendiev and M.R. Zachariah, Hybrid monte-carlo method for simulation of two-component aerosol coagulation and phase segregation, J. Colloid Inter. Sci., 49 (1) (2001), 30-43.

[9] Y. Efendiev and M.R. Zachariah, A model for two-component aerosol coagulation and phase separation: A method for changing the growth rate of nanoparticles, Chem. Eng. Sci, 56 (2001), 5763.

[10] S. Ehrman, M. Aquino-Class, and M.R. Zachariah, Effect of temperature and vaporphase encapsulation on particle growth and morphology, J. Materials Research, (1999), 1664-1671.

[11] S. Ehrman, S.K. Friedlander and M.R.Zachariah, Characterization of $\mathrm{SiO}_{2} / \mathrm{Ti}_{2}$ nanocomposite aerosol in a premixed flame, J. Aerosol Science, (1998), 687.

[12] S.K. Friedlander, Smoke, Dust and Haze, Oxford, (2000).

[13] A. Friedman and F. Reitich, Asymptotic behavior of solutions of coagulation-fragmentation models, Indiana Univ. Math. J., 47 (1998), 563-591. 
[14] D. Gillespie, An exact method for numerically simulating the stochastic coalescence process in cloud, J. Atmos. Sci., (1975).

[15] G. Jans, F.D.G. Lakshminarayanan, P. Lorentz and R. Tomkins, Molten Salts: Volume 1, Electrical Conductance, Density, and Viscosity Data, U.S. National Standard Reference Data Series, U.S. National Bureau of Standards, Washington, (1968).

[16] K.W. Lee, Change of particle size distribution during Brownian coagulation, J. Coll. Inter. Sci., (1983), 315-325.

[17] J.B. McLeod, On an infinite set of nonlinear differential equations, Quart. J. Math. Oxford Ser., 13 (1962), 119-128.

[18] B.K. McMillin, P. Biswas and M.R. Zachariah, In situ characterization of vapor phase growth of iron oxide-silica nanocomposite: Part I: 2-d planar laser-induced fluorescence and mie imaging. J. Mat. Res., (1996), 1552-156.

[19] E. Otto, F. Fissan, S. Park and K. Lee, Brownian coagulation in the transition regime using the moments of a lognormal distribution, J. Aerosol Sci., (1997), 629-630.

[20] E. Otto, E. Stratmann, F. Fissan, H. Vemury and S. Pratsinis, Brownian coagulation in the transition regime. 2: a comparison of two modeling approaches, J. Aerosol Sci., (1993), $535-536$.

[21] V. Piskunov and A. Golubev, Method for defining dynamical parameters of coagulating systems (in russian), Doklady Akademii Nauk, 366 (3) (1999), 341-344.

[22] M. Smith and T. Matsoukas, Constant-number Monte Carlo simulation of population balances, Chemical Eng. Science, (1998), 1777-1786.

[23] M. Smoluchowski, Drei vorträge über diffusion, brownsche bewegung und koagulation von kolloidteilchen, Physik. Z., (1916), 557-585.

[24] J. Spouge, Monte-Carlo results for random coagulation, J. Colloid Interface Sci., (1985), 38.

[25] P. Tandon and D. Rosner, Monte Carlo simulation of particle aggregation and simultaneous restructuring, J. Colloid Interface Sci, (1999), 273-276.

[26] M.R. Zachariah, M. Aquino-Class, R. Shull and E. Steel, Formation of superparamagnetic nanocomposite from vapor phase condensation in a flame, Nanostructured Materials, (1995), 383.

[27] M.R. Zachariah, R. Shull, B.K. McMillin and P. Biswas, In situ characterization and modeling of the vapor phase growth of a supermagnetic nanocomposite, Nanotechnology, (1996), 42-63. 